\title{
Green biotechnological approach as an alternative to chemical processes: the case of bioflocculant production through solid-state fermentation of soybean wastes
}

\begin{abstract}
Solid-state fermentation (SSF) from organic wastes can be considered as a novel biotechnological alternative for the development of new processes that may substitute the current chemical-dependence approaches. In this framework, the production of a bioflocculant through SSF using soybean fiber wastes as the sole substrate with the inoculation of Bacillus subtilis UPMB13 at different fermentation stages were carried out in a pilot scale near-to-adiabatic reactors of $4.5 \mathrm{~L}$ volume for 10 days. Samples collected at 2, 4, 7 and 10 days were checked for flocculating activity through kaolin assays and the crude bioflocculant was extracted through ethanol precipitation. The highest activities were measured at the 2 nd day of fermentation and maintained until the 4th day with an average value of 88.11792 .0 and $92.4132 .7 \%$ of bioflocculant activity for reactors inoculated at the beginning of SSF and after the thermophilic stage, respectively. The bioflocculant activities decreased around $3010 \%$ by the 7 th day. About $30 \mathrm{mg}$ of crude bioflocculant can be obtained from $20 \mathrm{~g}$ of sampled fermented substrate at the maximum flocculating activity measured. This SSF process can be considered as a new green alternative to produce bioflocculant from wastes under easily scalable conditions. Further studies may contribute to the industrial production of bioflocculants from wastes as an alternative to the commonly harmful commercial marketed flocculants.
\end{abstract}

Keyword: Bacillus subtilis UPMB13; Extracellular polymeric substance; Soybean fibers; Pilot scale 